\section{INFRARED NON-INVASIVE SUB-WAVELENGTH MICROSCOPY WITH METAMATERIALS}

Ward D. Newman

Electrical and Computer Engineering, University of Alberta
Eureka

Volume 3, Number 1 (2012)

\begin{abstract}
I demonstrate that hyperbolic metamaterials may provide the solution to the long-standing problem of the fundamental diffraction limit plaguing conventional microscopy and optical imaging systems. Presented here is the formalism of the classical electrodynamics used to describe the diffraction limit and sub-wavelength imaging using hyperbolic metamaterials. Effective medium theory is then derived for planar multilayer systems and put forth as a design method for such hyperbolic metamaterials; that is, multilayer systems consisting of alternating layers of metal and dielectric have an extreme anisotropy in the bulk dielectric tensor, leading to hyperbolic spatial dispersion. I then outline the design of a hyperbolic metamaterial base device for use in sub-wavelength infrared microscopy, and present numerical simulations to demonstrate the behaviour and performance of the device. The device employs multilayers of InGaAs/ AllnAs and is capable of sub-diffraction imaging resolution in the wavelength range of $8.8-10.5 \mu \mathrm{m}$. I show that high spatial frequency waves, which normally decay in vacuum, are allowed to propagate and reach the far-field in a hyperbolic metamaterial. Using a Green's function formalism to describe optical sources, sub-wavelength imaging capabilities of hyperbolic metamaterials is shown. Finally, potential device applications using the designed metamaterial are motivated.
\end{abstract}

\section{Introduction}

Microscopy and imaging has played a monumental role in modern science, and continues to do so. Biologists and many other fields of science have employed, what is now conventional optics, since at least the seventeenth century [1]. Even now, modern nano- and micro-fabrication facilities still rely heavily on basic to advanced microscopes for imaging and characterizing a wide range of objects such as integrated electrical circuits (ICs) and micro electromechanical devices (MEMs). In addition, one of the revolutionary micro-fabrication techniques, photolithography, relies on the physical principles of classical optics [2]. Nevertheless, there is still one major hurdle to overcome with optical microscopy and related technolo- gies, namely, the diffraction limit.

The diffraction limit is a physical effect which restricts the smallest feature size that can be resolved in an image to about half the imaging wavelength, $\sim \lambda / 2$. In terms of the optical spectrum ( $400-800 \mathrm{~nm})$, this limits the smallest resolvable feature size to $\geq 200 \mathrm{~nm}$. This physical phenomena is well explained using classical electrodynamics and Fourier optics; however, current technologies which can overcome this diffraction limit or which allow sub-wavelength (optical) resolution are either destructive (such as scanning electron microscopy and transmission electron microscopy) or invasive (atomic force microscope and scanning tunnelling microscope).

The emerging field of metamaterials may provide the solution to overcoming the diffraction limit through the ability to control and manipulate fundamental lightmatter interactions. This is achieved by tailoring the bulk and macroscopic material properties by controlling the sub-wavelength structure of a material. Twentieth century advances in nanofabrication have allowed the nanosctructure of devices to be tailored at will so that the fabrication of these metamaterials and their exotic electromagnetic properties are now possible [3]. In natural materials, the bulk and effective properties of a material is governed by the local arrangement (domain) of atoms, and the atomic lattice. A metamaterial is an artificially structured material with sub-wavelength structures known as meta atoms which govern the bulk electromagnetic properties as shown in figure 1.

In particular, Hyperbolic Metamaterials (HMMs) have shown great promise to overcoming the diffraction limit $[3,4]$ by allowing evanescent (decaying) EM waves which cannot be imaged using conventional optics, to propagate and hence be detected and imaged. The exotic property in HMMs which gives rise to this phenomena is an unbounded, hyperbolic dispersion relation. In natural transparent materials, the dispersion relation is bounded and spherical or ellipsoidal in shape.

Outlined here is the physics necessary to analyze the principles of sub-wavelength imaging and overcoming the diffraction limit, understanding the theory governing $\mathrm{HMMs}$, and designing and testing the performance of a HMM for use in the infrared (IR) region of the EM spectrum. The design of a HMM based device employing multilayers of InGaAs/AllnAs is summarized along with simulations of the performance of the device. These materials were first proposed for use in HMMs by Hoffman [5], however the scope of that work was on negative refraction; therefore, the theoretical and design developed and presented here, for applications in sub-wavelength imaging, is new and state-of-the-art.

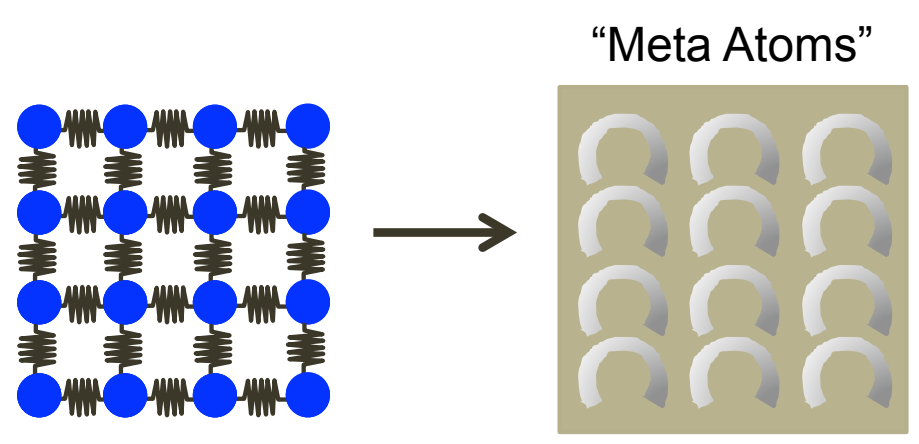

FIG. 1 In natural materials, the bulk EM properties are governed by the arrangement of atoms. In metamaterials, the bulk EM properties are governed by artificially structured "Meta atoms". 


\section{Sub-Wavelength Imaging in the IR}

Plane Wave Propagation in Anisotropic Uniaxial Media

Maxwell's equations for the time dependent electric and magnetic fields can be decoupled to yield the wave equations for EM waves, that is light in a sourceless region

$$
\left(\nabla \times \nabla \times+\frac{\overline{\mu \epsilon}}{c^{2}} \frac{\partial^{2}}{\partial t^{2}}\right)\left\{\begin{array}{l}
\vec{E} \\
\vec{B}
\end{array}\right\}=0
$$

where $\mathrm{c}$ is the speed of light, and $\bar{\mu}$ and $\bar{\epsilon}$ are the relative magnetic permeability and electric permittivity tensors respectively.

In the optical and IR region of the EM spectrum, almost all materials do not have a magnetic response so it is safe to assume $\bar{\mu}=\bar{I}$, the identity tensor. In addition, if the medium is uniaxial, then the dielectric tensor is of the form

$$
\bar{\epsilon}=\left(\begin{array}{ccc}
\epsilon_{\|} & 0 & 0 \\
0 & \epsilon_{\|} & 0 \\
0 & 0 & \epsilon_{\perp}
\end{array}\right)
$$

where the dielectric constant along the $x$-and $y$-directions is $\varepsilon_{x}$, and the dielectric constant along the $z$-direction is $\varepsilon_{z}$. This type of material has a planar symmetry in the xy-plane and the dielectric tensor has two unique components, namely $\varepsilon_{\|}$in the xy-plane and $\varepsilon_{\perp}$ in the direction normal to the $x y$-plane.

These wave equations give rise to plane wave solutions of the form

$$
\left\{\begin{array}{l}
\vec{E}(\vec{r}, t) \\
\vec{B}(\vec{r}, t)
\end{array}\right\}=\left\{\begin{array}{l}
\vec{E}_{0} \\
\vec{B}_{0}
\end{array}\right\} e^{i \vec{k} \cdot \vec{r}} e^{-i \omega t}
$$

provided that the propagation wavevector $\vec{k}$, satisfies the equation [6]

$$
\vec{k} \times\left(\vec{k} \times \vec{E}_{0}\right)=\left(\frac{\omega}{c}\right)^{2} \bar{\epsilon} \vec{E}_{0}=k_{0}^{2} \bar{\epsilon} \vec{E}_{0}
$$

This describes a wavevector surface from which the details of propagation of different types of $\vec{E}_{0}$ polarizations in the media are obtained.

From equations 3 and 4 , it then follows that for uni-axial materials described by equation 2, there are two unique types of plane waves described in equation 3: (a) Where $\vec{E}_{0}$ is parallel to the $x y$-plane and (b) where $\vec{B}_{0}$ is parallel to the $x y$-plane. These correspond to transverse electric TE polarizations (ordinary waves) and transverse magnetic polarizations TM (extraordinary) respectively. The relation between $\vec{E}_{0}$ and $\vec{B}_{0}$ for each case are easily obtained

through Maxwell's Equations: $\nabla \times \vec{E}=-\frac{1}{c} \frac{\partial \vec{B}}{\partial t}$ and $\nabla \times \vec{B}=\frac{\bar{\epsilon}}{c} \frac{\partial \vec{E}}{\partial t}$. For the remainder of this discussion, only TM polarization will be treated, however, analogous arguments and derivations can performed for TE waves.

For TM polarized light, equation 4 reduces to the well known dispersion, or isofrequency relation for extraordnary waves in an uniaxial medium

$$
\frac{k_{x}^{2}+k_{y}^{2}}{\epsilon_{\perp}}+\frac{k_{z}^{2}}{\epsilon_{\|}}=k_{0}^{2}
$$

with $k_{0}=2 \pi / \lambda=\omega / c$.

\section{Diffraction Limit}

Vacuum and most natural dielectrics (glasses) are not uniaxial, but isotropic so that $\epsilon_{\|}=\epsilon_{\perp}=\epsilon>0$

Equation 5 then becomes

$$
k_{x}^{2}+k_{y}^{2}+k_{z}^{2}=\epsilon k_{0}^{2}
$$

and the wave vector surface described by equations 5 and 6 is a sphere ${ }^{1}$. In terms of imaging,we wish for an object in the $x y$-plane to be focused and imaged in the $x^{\prime} y^{\prime}$-plane, some distance $z$ from the object, figure 3. From Fourier Optics, the electric field corresponding to the object in the $x y$-plane (WLOG assume $z=0$ ) can be decomposed into plane waves (spatial frequencies) $k_{x}$ and $k_{y}$ through a Fourier transform into a Fourier (or angular) spectrum as [7]

$$
\begin{gathered}
\widehat{E}\left(k_{x}, k_{y}, z=0\right)= \\
\iint_{-\infty}^{+\infty} E(x, y, z=0) e^{i\left(k_{x} x+k_{y} y\right)} d x d y
\end{gathered}
$$

This Fourier plane wave spectrum propagates along the z-axis (imaging axis) as [7]

$$
\widehat{E}\left(k_{x}, k_{y}, z\right)=\widehat{E}\left(k_{x}, k_{y}, z=0\right) e^{i k_{z} z}
$$

where $k_{z}$ is derived from from the isofrequency relation,

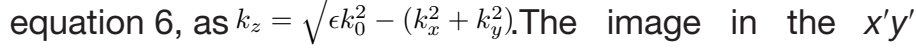
plane (at $z=z>0$ ) then corresponds to the inverse Fourier transform of this spectrum (eq 8)

$$
\begin{aligned}
& \vec{E}(x, y, z)= \\
& \quad \int_{-\infty}^{+\infty} \widehat{E}\left(k_{x}, k_{y}, z=0\right) e^{i\left(k_{x} x+k_{y} y\right)} e^{i k_{z} z} d k_{x} d k_{y}
\end{aligned}
$$

However, from equation 6 we see that for large $k_{x}$ or $k_{y}$, then $k_{z}$ is complex, and the $e^{i k_{z} z}$ term in equation 9 is exponentially damping; these waves are evanescent and decay before reaching the far-field (fig 5a). That is, the high spatial frequencies necessary to make up the sharp features of the object, do not propagate and therefore cannot be detected nor imaged in the far-field ${ }^{2}$. This is known as the diffraction limit and this concept is illustrated in figure 4.

The maximum magnitude of spatial frequency wavevector allowed to propagate and hence contribute to a far field image is given by equation 6 and is written explicitly as

$$
k_{\max } \leq \sqrt{\epsilon} k_{0}=n k_{0}
$$

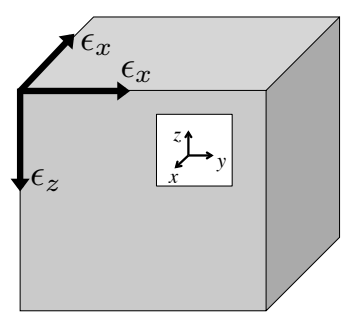

FIG. 2. Schematic of the dielectric tensor for a uniaxial medium. There is a frequencies making up the electric field planar symmetry in the xy-plane so that of an aperture or object in the $\varepsilon_{\mathrm{x}}=\varepsilon_{\|}$, and $\varepsilon_{\mathrm{z}}=\varepsilon_{\perp}$.

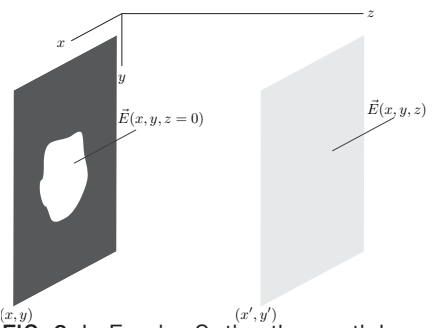

FIG. 3. In Fourier Optics the spatial $(x, y, z=0)$ plane are propagated to the imaging plane $\left(x^{\prime}, y^{\prime}\right)$.

1. For the case of uniaxial crystal propagation, both $\varepsilon_{\|}>0$ and $\varepsilon_{\perp}>0$, so that the wavevector surface is an ellipsoid, and the remaining diffraction limit arguments still apply but must be modified slightly.

2. Far field refers to an image plane with $z>\lambda$ (image field). 


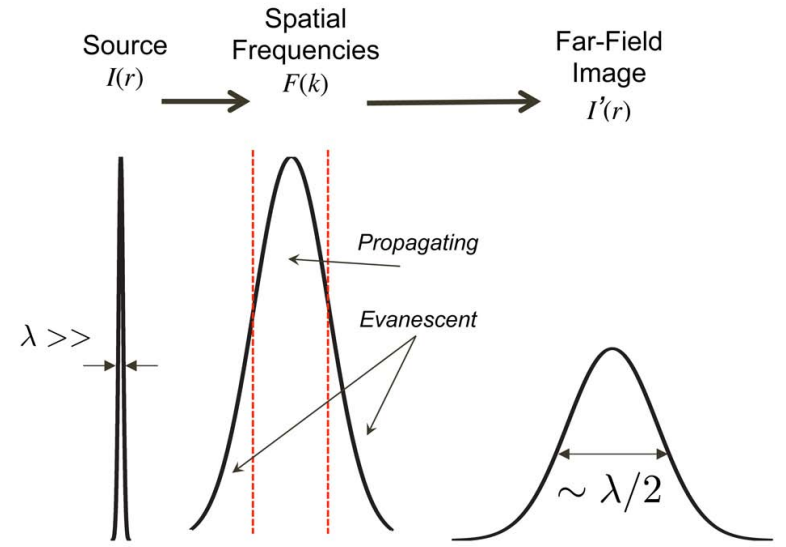

FIG. 4. A highly localized EM source, object, or an object with sharp features has a broad spatial frequency spectrum (fourier spectrum). The high spatial frequencies correspond to evanescent plane waves which decay and do not reach the farfield. As a result, the reconstructed image has lost information, and any original feature smaller than about $\lambda / 2$ is blurred. Furthermore, objects closer than $\sim \lambda / 2$ to each other can not be resolved using traditional optics.

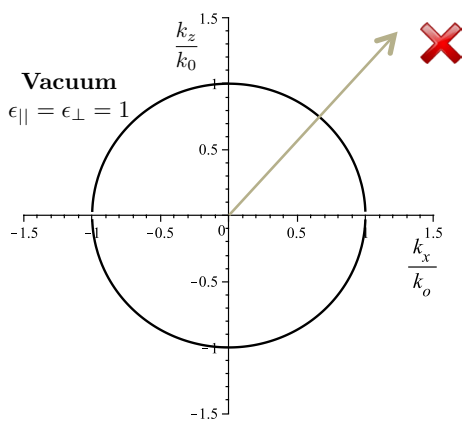

(a) Vacuum

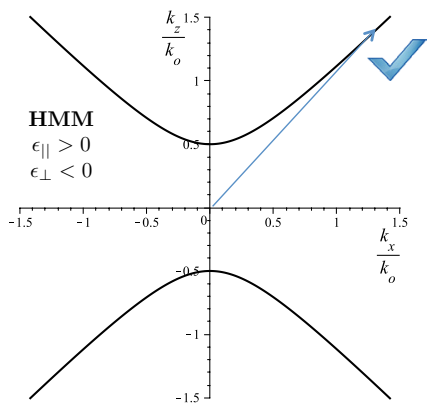

(b) HMM
FIG. 5. (a) In Vacuum and natural dielectric materials, high spatial frequency modes are not supported and are evanescent. High spatial frequencies (large $k_{x}$ ) above $\varepsilon \mathrm{k}_{0}$ yield imaginary propagating wavevectors $\left(\mathrm{k}_{\mathrm{z}}\right)$, and these components of the Fourier (angular) spectrum of an object do not reach the far field (image field). (b) HMMs have a hyperbolic dispersion relation which supports all spatial frequencies. This implies that the whole Fourier or Angular spectrum is propagated to the far field, making HMMs ideal for subwavelength imaging appliations.

This corresponds to a minimum resolvable feature size of $d \approx 2 \pi k_{\max }^{-1}=\lambda / n$. For vacuum, $\mathrm{n} \approx 1.5$ and the minimum resolvable feature size is $\mathrm{d} \approx \lambda / 2$.

\section{Beat the diffraction Limit}

There are several ways to obtain the near field information (high spatial frequencies) of an object such as atomic force microscopy (AFM), or a near field scanning optical microscopy (NSOM). However, these methods are invasive as they require that a detector (usually a very fine tipped needle) be placed very close $(<<\lambda)$ to the object to be imaged [2].Therefore, these sub-diffraction imaging techniques are not suitable for many type of samples.

Other methods of obtaining very high resolution images are scanning electron microscopy (SEM) and transmission electron microscopy (TEM) [2]. These methods utilize the wave nature of electrons (through the de Brogolie relation), and therefore are subject to the diffraction limit as well, however the characteristic wavelength of the electrons can be made much smaller than that of optical and IR light. As a result, the smallest resolvable feature size is smaller than that obtained with conventional optical techniques. However, SEM and TEM tend to be destructive to the sample.

Metematerials provide a new non-destructive/invasive route to allowing these high spatial frequency modes, which normally decay in vacuum and natural materials, to propagate and reach the far field, allowing them to be sensed and imaged.

In the following section, I present a new type of metamaterial, Hyperbolic Metametierials, which allows all spatial frequency modes to propagate into the far field.

\section{HYPERBOLIC METAMATERIALS}

Hyperbolic Metamaterials (HMMs) are an artificially structured material with a dielectric tensor with an extreme anisotropy.

In short, HMMs behave like a metal (with $\varepsilon<0$ ) in one direction and like a dielectric $(\varepsilon>0)$ in another. Planar HMMs are uniaxial and the dielectric tensor has the form

$$
\bar{\epsilon}_{H M M}=\left(\begin{array}{ccc}
\epsilon_{\|} & 0 & 0 \\
0 & \epsilon_{\|} & 0 \\
0 & 0 & \epsilon_{\perp}
\end{array}\right)
$$

with $\varepsilon_{\|}>0$ and $\varepsilon_{\perp}>0$.

This extreme anisotropy yields the exotic behaviour of HMMs: hyperbolic dispersion. With this type of extreme anisotropy, equation 5 yields a wavevector surface of a hyperboloid.

$$
-\frac{k_{x}^{2}+k_{y}^{2}}{\left|\epsilon_{\perp}\right|}+\frac{k_{z}^{2}}{\epsilon_{\| \mid}}=k_{0}^{2}
$$

Using this relation along with the Fourier spectrum arguments made in section II B, all high spatial frequencies are allowed to propagate inside an HMM.That is, for any magnitude of $\sqrt{k_{x}^{2}+k_{y}^{2}}$, there is always a real $k_{z}$, and the whole Fourier or Angular spectrum is propagated into the far field [3]. Therefore, HMMs have a strong potential for sub-wavelength imaging in the far field, that is the ability to beat the so called diffraction limit. The isofrequency curves for an ideal HMM and vacuum are shown in figure 5.

HMMs are not found naturally in nature so must be designed and fabricated to have the required bulk or effective medium properties. Advances in nanofabrication have allowed the nanoscopic structure of materials to be tailored at will, allowing bulk HMM properties to be observed for applications in imaging [4]. In the Effective Medium Theory section below, a novel technique to realize a bulk planar medium with an extreme anisotropy characteristic of HMMs is outlined and discussed.

\section{HMM Design: Effective Medium Theory Introduction}

If the structure of an object is much smaller than the wavelength of light interacting with the object, then the response of EM radiation interacting with the structure can be characterized by a bulk or effective medium. Information acquired through measurements on the incident, reflected, and transmitted light will yield properties of an effective, homogeneous medium, not the properties of the media making up the sub-wavelength structure.

Effective medium theory (EMT) or effective medium approximation is a technique to average these subwavelength properties, and predict the bulk response of EM radiation interacting with the structure. Presented in detail 
below is an effective medium theory for planar multilayer structures, and a novel way for designing HMM behaviour in a metal/dielectric multilayer structure.

\section{Effective Medium Theory for Multilayer Structures}

Consider the multilayer structure shown in figure 6 in which the wavelength of the interacting electromagnetic wave $\lambda$ is much larger than the layer thicknesses $d_{1}$ and $d_{2}$. For brevity and simplicity assume $d_{1}=d_{2}=d$. The derivation for the more general case is analogous and will be stated later. The electric flux density in each medium is related to the electric field as

$$
\vec{D}=\bar{\epsilon} \vec{E}
$$

where $\bar{\epsilon}$ is the dielectric tensor. Let each medium on its own be isotropic so that the dielectric tensor can be represented by a dielectric constant, say $\bar{\epsilon} \rightarrow \epsilon_{1}$ for medium 1 and $\bar{\epsilon} \rightarrow \epsilon_{2}$ for medium 2. Therefore, $\overrightarrow{D_{1}}=\epsilon_{1} \overrightarrow{E_{1}}$ and $\overrightarrow{D_{2}}=\epsilon_{2} \overrightarrow{E_{2}}$ Since the wavelength of light is much larger than the layer thickness $\left(\lambda>>\right.$ d) then $\overrightarrow{D_{1}}$ does not vary over several layers of medium 1 , and similarly, $\overrightarrow{D_{2}}$ does not vary over several layers of medium 2,then it follows that the average value of $\vec{D}$ throughout the structure can be determined by analyzing the relation between $\overrightarrow{D_{1}}$ and $\overrightarrow{D_{2}}$ at one interface between medium 1 and medium 2. The average value of $\vec{n}$ will then be related to the average electric field by the effective dielectric tensor

$$
<\vec{D}>=\frac{\vec{D}_{1}+\vec{D}_{2}}{2}=\bar{\epsilon}_{\text {eff }}<\vec{E}>
$$

with $<\vec{E}>=\left(\vec{E}_{1}+\vec{E}_{2}\right) / 2$. The electromagnetic boundary conditions at an interface between medium 1 and medium 2 can be derived from Maxwell's Equations [8]

$$
\begin{aligned}
D_{1}^{\perp} & =D_{2}^{\perp} \\
\vec{E}_{1}^{\|} & =\vec{E}_{2}^{\|}
\end{aligned}
$$

By employing the second of the two above boundary conditions, the average electric flux density parallel to the interface is

$$
\begin{aligned}
<\vec{D}^{\|}> & =\frac{\vec{D}_{1}^{\|}+\vec{D}_{2}^{\|}}{2}=\frac{\epsilon_{1} \vec{E}_{1}^{\|}+\epsilon_{2} \vec{E}_{2}^{\|}}{2} \\
& =\frac{\epsilon_{1}+\epsilon_{1}}{2}<\vec{E}^{\|}>
\end{aligned}
$$

where equation 16 implies $\vec{E}_{1}^{\|}=\vec{E}_{2}^{\|}=<\vec{E}^{\|}>$. The parallel component of the effective dielectric tensor is arithmetic mean of the two dielectric constants of the two media.

Similarly, employing the first of the two boundary conditions, the average electric flux density perpendicular to the interface is computed as

$$
\begin{aligned}
<D^{\perp}> & =\frac{D_{1}^{\perp}+D_{2}^{\perp}}{2}=\frac{\epsilon_{1} E_{1}^{\perp}+\epsilon_{2} E_{2}^{\perp}}{2} \\
& =\frac{2 \epsilon_{1} \epsilon_{1}}{\epsilon_{1}+\epsilon_{2}}<E^{\perp}>
\end{aligned}
$$

where equation 15 was used to relate $E_{1}^{\perp}$ and $E_{2}^{\perp}$.

The perpendicular component of the effective dielectric tensor is the harmonic mean of the two dielectric constants of the two media.

Therefore, the effective dielectric tensor is anisotropic and can then be written as

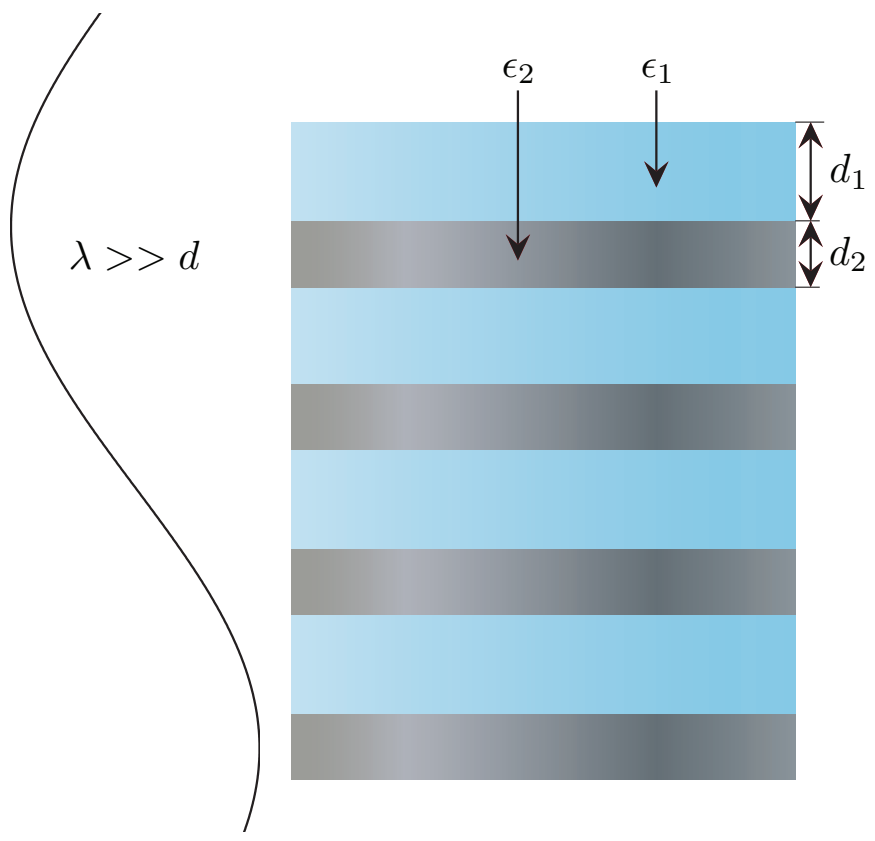

FIG. 6. Multilayer stack used for effective medium theory analysis.

$$
\bar{\epsilon}_{\mathrm{eff}}=\left(\begin{array}{ccc}
\epsilon_{\|} & 0 & 0 \\
0 & \epsilon_{\|} & 0 \\
0 & 0 & \epsilon_{\perp}
\end{array}\right)=\left(\begin{array}{ccc}
\frac{\epsilon_{1}+\epsilon_{1}}{2} & 0 & 0 \\
0 & \frac{\epsilon_{1}+\epsilon_{1}}{2} & 0 \\
0 & 0 & \frac{2 \epsilon_{1} \epsilon_{1}}{\epsilon_{1}+\epsilon_{2}}
\end{array}\right)
$$

where the parallel components lie in the $x y$-plane and the perpendicular direction is considered the $z$-direction.

When $d_{1} \neq d_{2}$, the above can generalized to

$$
\begin{gathered}
\epsilon_{\|}=\rho \epsilon_{2}+(1-\rho) \epsilon_{1} \\
\frac{1}{\epsilon_{\perp}}=\frac{\rho}{\epsilon_{2}}+\frac{1-\rho}{\epsilon_{1}}
\end{gathered}
$$

with the weighting factor $\rho=\frac{d_{2}}{d_{1}+d_{2}}$.

This shows that a general anisotropic uniaxial medium can be fabricated from a periodic multilayer stack with the layer thicknesses much smaller than the desired design EM wavelength. More subtly, however, the thickness of the layers should be chosen so that the quantization of energy levels in the system is irrelevant [5].

\section{Effective Medium Theory For HMM Design}

Referring to figure 6 and equations 20 and 21, if material 1 is a metal and material 2 is a dielectric $\left(\varepsilon_{\|}<0\right.$ and $\left.\varepsilon_{\perp}<0\right)$, then the effective medium dielectric constants are also of opposite signs. That is,

$$
\text { If }\left\{\begin{array} { l } 
{ \epsilon _ { 1 } < 0 } \\
{ \epsilon _ { 2 } > 0 }
\end{array} \text { then } \left\{\begin{array} { l } 
{ \epsilon _ { \| } < 0 } \\
{ \epsilon _ { \perp } > 0 }
\end{array} \text { or } \left\{\begin{array}{l}
\epsilon_{\|}>0 \\
\epsilon_{\perp}<0
\end{array}(22)\right.\right.\right.
$$

which is the exact extreme anisotropy required for HMM behaviour.

Therefore, a simple multilayer structure with alternating layers of dielectric and metal has the bulk EM response required for a HMM.

\section{Design Results: HMM in IR Materials}


For HMM design in the IR region we require a metallic material and a dielectric material to employ the EMT design technique outlined in the previous section.

An obvious choice for the metallic material would be a traditional metal (Ag, Au, etc), however, in the IR losses are much too high. Another possible choice is silicon carbide, $\mathrm{SiC}$, which displays metallic behaviour in the IR [9], however, the dispersion is much too rapid and the losses in the material are significant, making a desired broadband EMT design for imaging applications ineffective. For this design, InGaAs was chosen as it displays metallic behaviour in the IR which can be tailored at will with Si doping. The losses in InGaAs are also relatively low.

As outlined by Hoffman [5], the dielectric constant for InGaAs can be computed using the Drude Model (free electron oscillator) for metals

$$
\epsilon_{\text {InGaAs }}(\omega)=\epsilon_{\infty}\left(1-\frac{\omega_{p}^{2}}{\omega^{2}-i \omega / \gamma}\right)
$$

where $\varepsilon_{\infty}=12.15$ is the background dielectric constant, and $\gamma=0.1 \times 10^{-12} \mathrm{~s}^{-1}$ represents losses in the system and reflects the collision rate of electrons with atoms. The plasma frequency $\omega_{\mathrm{p}}$ can be tailored through Si doping: a donor atom such as phosphorus diffuses into $\mathrm{Si}$ and replaces an Si atom in the regular Si crystal lattice; each donor donates a free electron capable of conducting electricity. If the concentration of donor atoms is $n_{\mathrm{d}}$, then the plasma frequency is given in the Drude model as

$$
\omega_{p}^{2}=\frac{n_{d} e^{2}}{\epsilon_{0} m_{e}^{*}}
$$

where $e$ is the free electron charge, $\varepsilon_{0}$ is the permittivity of free space, and $m_{e}^{*}$ is the effective mass of electrons in InGaAs. Under moderate EM intensities and a doping density of $8 \times 10^{18}$ donors $/ \mathrm{cm}^{3}$, the effective electron mass in InGaAs is $m_{e}^{*} \approx 0.53 m_{e} \quad$ [2] and the plasma frequency is evaluated as $\omega_{p}=2.2 \times 10^{14} \mathrm{~s}^{-1}$

For the dielectric, AllnAs was chosen as it displays very little dispersion in the IR and negligible losses. For most of the IR spectrum $\epsilon_{\mathrm{AIInAs}}=10.23 \quad$. Figure 7 shows the simulated dispersion of the dielectric constants for AllnAs and InGaAs in the IR.

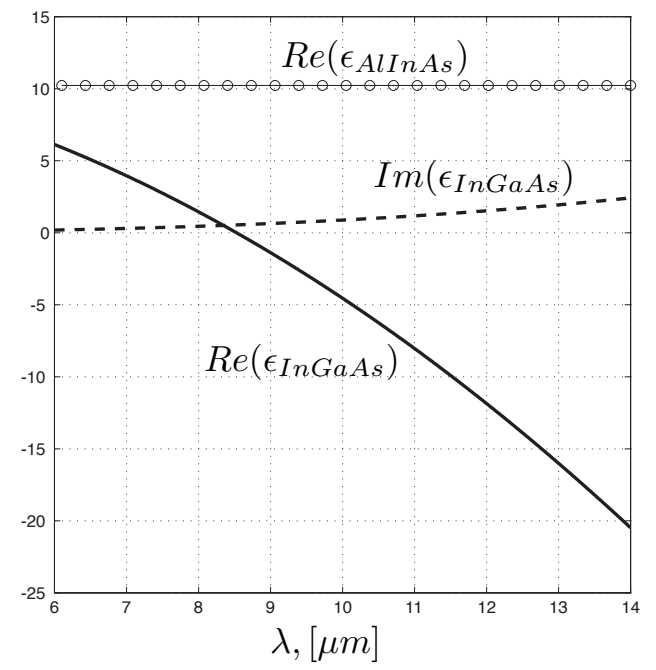

FIG. 7. AllnAs shows very little dispersion in the IR and remains $\approx 10.23$. The real and imaginary parts of $\varepsilon$ InGaAs is computed using the Drude Model for metals at a Si doping level of $8 \times 10^{18} \mathrm{~cm}^{-3}$.
Furthermore, highly crystalline films of these materials can be grown using molecular beam epitaxy.

Since AllnAs/InGaAs are lattice matched, the multilayer structure will have a very low intrinsic stress, and the layer interfaces will be very smooth results in ideal fresnel reflection and transmission [5].

Effective Medium Dielectric Tensor

Using the effective medium design techniques outlined above (equations 20 and 21), along with the material properties derived in the previous section, the effective medium constants $\left(\varepsilon_{\|}\right.$and $\left.\varepsilon_{\perp}\right)$ for a multilayer stack of InGaAs/AllnAs were computed and optimized.

To minimize reflections (with imaging in mind), we require $\varepsilon_{\|} \sim 1$. Therefore, I selected $\varepsilon_{\|}=1$ at a wavelength of $\lambda=10 \mu \mathrm{m}$, roughly the centre wavelength of the desired operation range. In addition, to reduce the total number of layers needed while keeping $\mathrm{d}<<\lambda$, I chose $\mathrm{d}_{\text {AllnAs }}=80 \mathrm{~nm}$. Then from equation 20, the InGaAs thickness is computed as $d_{\text {AllnAs }}=130.5 \mathrm{~nm}$. With reference to equations 20 and 21, this corresponds to a weighting factor $\rho=0.62$.

Using these layer thicknesses, equations 20 and 21, and the dielectric constants of the InGaAs and AllnAs computed in section VA, the effective dielectric tensor was calculated. From this simulation, it was determined that the AllnAs/InGaAs multilayer displayed HMM behaviour in the wavelength region $\$ 8.8-10.5 \mu \mathrm{m}$, for a total bandwidth of $\Delta=1.7 \mu \mathrm{m}$. However, starting $\sim 10.3 \mu \mathrm{m}$ losses begin to become significant, and propagation lengths inside the bulk material (overall multilayer structure) would diminish. Figure 8 shows the wavelength dispersion of $\varepsilon_{\|}$and $\varepsilon_{\perp}$.

\section{Simulation of HMM Behaviour At A Single Spatial Frequency}

The very exotic and useful behaviour of HMMs for applications in sub-wavelength imaging is the fact that normally evanescent modes (high spatial frequencies) are allowed to propagate inside an HMM.

To demonstrate this behaviour, I simulated an evanescent, decaying, plane wave incident upon a slab of HMM (effective medium theory $\varepsilon_{\|}$and $\varepsilon_{\perp}$, and an evanescent wave incident upon the exact multilayer structure $\left(\varepsilon_{\text {InGaAs }}\right.$, $\varepsilon_{\text {AllnAs }}$ ).

Using the fresnel reflection coefficients for TM polarized light incident upon a slab of anisotropic medium (see Fowles [6] for example ), and the expressions for the plane waves developed in section IIA the intensity of an evanescent plane wave $(\lambda=9.8 \mu \mathrm{m})$ incident upon a slab HMM with the effective medium constants shown in figure $\backslash$ ref\{HMMDispersion\} was modeled. The simulation confirmed the expected bulk behaviour of the HMM; namely, evanescent waves are captured by the HMM and are allowed to propagate into the far field. Figure 9 illustrates this result. The figure shows the field profile as the decaying wave propagates in the $\mathrm{z}^{+}$-direction; the $\mathrm{x}$ and $y$-directions have been supressed. We see nearly a 10X increase in the signal strength at the exit side of the HMM slab as compared to vacuum.

To test the validity of the effective medium design and simulation techniques used, a plane wave incident upon the exact InGasAs/AllnAs multilayer structure was modelled. 


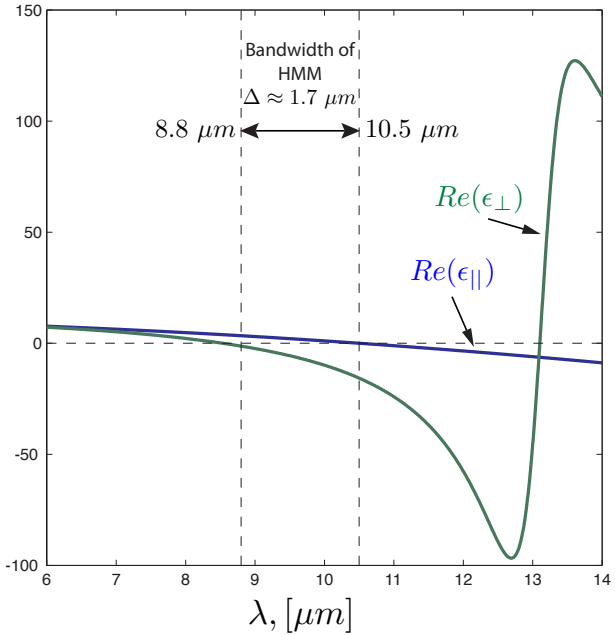

FIG. 8. Dispersion of effective dielectric constants, $\varepsilon_{\|}$ and $\varepsilon_{\perp}$ for AllnAs/InGaAs $(80 \mathrm{~nm} / 130.5 \mathrm{~nm})$ multilayer structure. Multilayer has HMM behaviour when $\varepsilon_{\|}>0$ and $\varepsilon_{\perp}<0(8.8-10.5 \mu \mathrm{m})$.

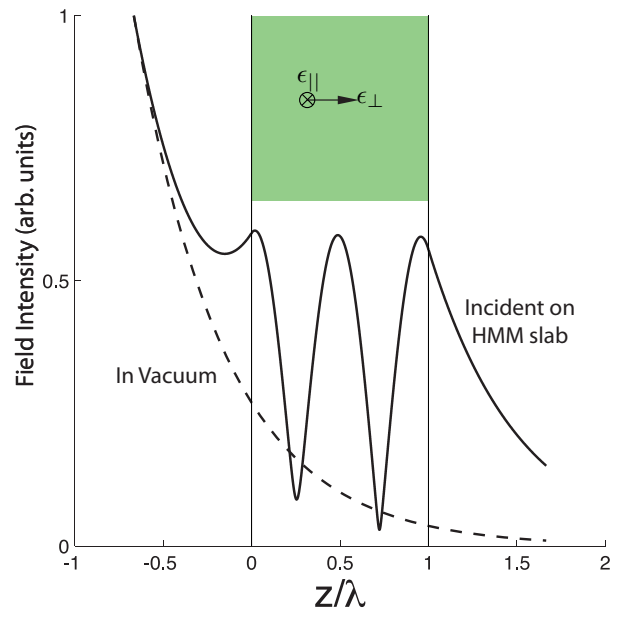

FIG. 9. Evanescent, high spatial frequency waves which decay in vacuum are captured by the HMM slab and allowed to propagate to the far field.

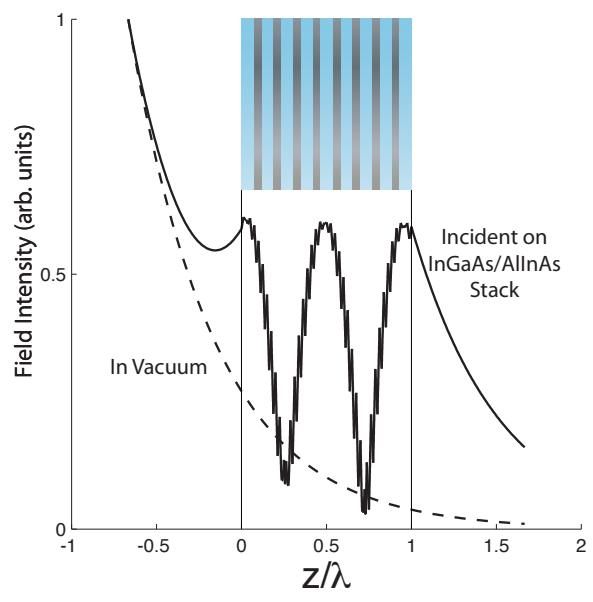

FIG. 10. Exact numerical simulation of the AllnAs/ InGaAs multilayer structure. The bulk response of the multilayer structure agrees very closely with that predicted by effective medium theory. The evanescent, high spatial frequencies waves propagate to the far field through the multilayer structure.
Using a transfer matrix method for obtaining the propagation amplitude constants within multilayer structures (see Yeh [10] for for derivation and discussion of transfer matrix technique), and the form of the fields for plane waves developed in section IIA, the intensity of an evanescent plane wave incident upon the designed AllnAs/ InGaAs multilayer stack was simulated. As shown in figure 10 , the simulation shows the fact that although there is very subtle physics governing the individual layers ${ }^{3}$, the bulk response of the multilayer structure agrees very closely with that observed for the effective medium slab, therefore confirming the validity and accuracy of the effective medium technique for designing HMMs.

We now have confirmation that the multilayer of InGaAs/ AllnAs does posses the exotic HMM behaviour required for sub-diffraction imaging. Below, I show that sub-wavelength resolution of a radiating point source such as an excited $\mathrm{C}-\mathrm{O}$ bond can be obtained in the far-field by utilizing a InGaAs/AllnAs metameterial.

\section{Green's Function Formalism of Scattering and Emission: Sub-wavelength Imaging Introduction}

Scattering, diffraction, and emission centers can be modelled using a radiating point dipole. The details of the near-field (the propagating and evanescent modes), must be obtained through the Green's function formalism of EM sources.

To demonstrate sub-wavelength imaging, I simulated the emitted electric field intensity of a point dipole above a slab of the designed effective medium AllnAs/InGaAs HMM.

3. The high $k_{x}$ evanescent waves excite surface plasmons polaritons on the metal layers, a collective oscillation of electrons within a metallic layer. These surface plasmons then couple together between metallic layers to yield a collective response and allow the evanescent wave to propagate. See Maier's book for an in depth discussion of this phenomena[11].

\section{Derivation and Results}

If there is a localized current source $\vec{J}(\vec{r})$ in vacuum then if we assume time harmonic fields (i.e. all fields and the current source oscillate in time at a frequency $\omega$ ), Maxwell's wave equation for the resulting electric field becomes

$$
\left[(\nabla \times \nabla \times)-k_{0}^{2}\right] \vec{E}=i \frac{4 \pi k_{0}}{c} \vec{J}
$$

again with $k_{0}=\omega / c$. This is the well known vector Helmholtz equation.

Now, a point dipole located at the position $\vec{r}=\vec{r}_{0}$ can be described by a point current source $\vec{J}(\vec{r})=\vec{\mu} \delta\left(\vec{r}-\vec{r}_{0}\right.$ where $\vec{\mu}$ gives the direction of the dipole (current source).

With this point current source, Maxwell's wave equation can be solved using a Green's Function Technique [7].

The derivation is quite in depth and only the results will be presented here. For simplicity, the dipole is oriented normal to the slab; in this way, all the EM radiation incident on the HMM is TM polarized [7]. By employing the Green's Function Technique for solving the vector Helmholtz equation, the general form for the fields emitted by a radiating point dipole oriented along the $z$-axis $(\vec{\mu}=[0 ; 0 ; 1])$ and at a position $z=d$ can be shown to be

$$
\vec{E}=\left[\widehat{D} \iint_{-\infty}^{+\infty} \frac{1}{k_{z}} e^{i\left(k_{x} x+k_{y} y+k_{z}|z-d|\right)} d k_{x} d k_{y}\right] \cdot\left(\begin{array}{l}
0 \\
0 \\
1
\end{array}\right)
$$

where the differential operator $\widehat{D}$ being

$$
\widehat{D}=\left(\bar{U}+\frac{1}{k_{0}^{2}} \vec{\nabla} \vec{\nabla}\right)
$$

and

$$
\bar{U}=\left(\begin{array}{lll}
1 & 0 & 0 \\
0 & 1 & 0 \\
0 & 0 & 1
\end{array}\right)
$$

The double integral term represents the angular spectrum or plane wave representation of a scalar point source emitting spherical waves.

The Dyadic operator $\widehat{D}$, acting on this integral essentially 
vectorizes and transforms the spherical point source into electric fields corresponding to the orientation of the dipole vector $\vec{\mu}=(0 ; 0 ; 1)$.

The double integral in equation 26 is very computationally heavy due to the limits of integration, and moreover, because there is a singularity when $k_{z}$ changes from real to imaginary (when the spatial frequencies become evanescent).

It is numerically convenient to then convert this 2-D integral which ranges over $\left(k_{x}, k_{y}\right)$ into a 1-D integral which runs over $k_{r}$ through a cylindrical coordinate transform, and in addition, to split the integrals up into propagating and evanescent parts [12]. The forms of the decomposed integrals are then the well known numerical "Sommerfeld Integrals".

Because the form of the double integral in equation 26 is that of a summation of plane waves $\sim e^{i\left(k_{x} x+k_{y} y+k_{z} z\right)}$ with amplitudes $1 / k_{z}$ then it is possible to obtain the forms of the reflected and transmitted electric fields, $\vec{E}_{R}$ a $\mathrm{n} \mathrm{d} \vec{E}_{T}$ using the fresnel reflection and transmission coefficients at a single spatial frequency pair $\left(k_{x}, k_{y}\right)$. Similarly, the resulting integrals for $\vec{E}_{R}$ and $\vec{E}_{T}$ can then be converted to Sommerfeld integrals.

Using the tools derived above, the fields of a monochromatic radiating dipole $(\lambda=9.8 \mu \mathrm{m})$ were then simulated in MATLAB for two cases.

First, when the dipole was isolated in vacuum, and second when the dipole was brought near the effective medium HMM of the InGaAs/AllnAs multilayer.

The results of these simulations are shown in figure 11.

In vacuum the high spatial frequency modes required for imaging the very small features of the source decay quickly and the over all emission is essentially non-directional. As the source is brought close to the HMM, the high spatial frequency modes couple into the HMM resulting in highly directional emission. This very important result shows that very high resolution information may be carried to the farfield. The implications of this extremely exotic behaviour are discussed in the section below.

\section{Device Implications and Design}

The planar HMM designed in this project has a wide range of applications from a bio-analytical tools to integrated circuit quality control.

Each chemical species emits a unique IR fingerprint due to characteristics of the vibrations in the chemical bonds. This allows for spectroscopy to identify specific chemical
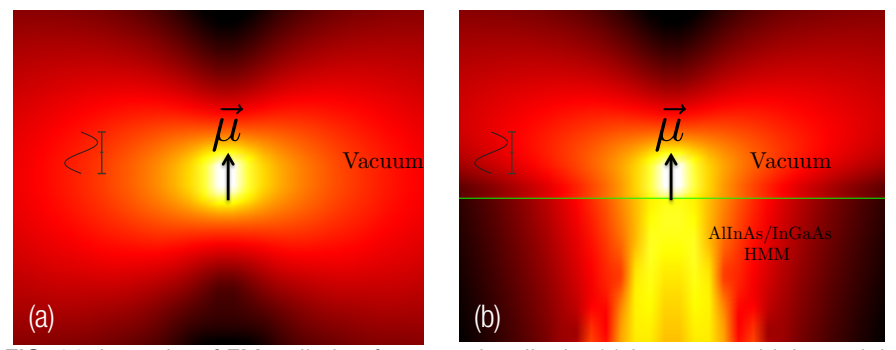

FIG. 11. Intensity of EM radiation from a point-dipole. (a) In vacuum, high spatial frequency modes decay and emission is non-directional. (b) When dipole is brought near HMM, the high spatial frequency, evanescent modes couple into HMM resulting in highly directional emission. High spatial frequencies reach far field. species, based on the observed IR spectrum.Combining spectroscopy with microscopy allows for drug permeation in human tissue to be observed (fig 12).

By using HMM sub-wavelength imaging techniques, it may be possible to observe drug permeation and chemical activity within a single cell. The characteristic size of cells is much smaller than that of tissue, so to resolve these sub-wavelength (IR) features, sub-wavelength imaging techniques are required. This type of application has incredible importance especially in the pharmaceutical industry, and in the Bio-MEMs industry.

$\mathrm{Si}$ is transparent to IR wavelengths; therefore, cracks or defects in ICs or MEMs can be detected through the scattering and diffraction of IR light at these defect centres [14]. The main problem with the conventional technique is the diffraction limit: cracks and defects smaller than the illuminating wavelength can not be imaged. In terms of IR this corresponds to $\sim 2-3 \mu \mathrm{m}$. By using a HMM, subwavelength resolution may be obtained so that defects with sub-wavelength dimensions may be observed and detected. This type of application/ device is conceptualized in figure 13.

\section{Conclusion}

I have motivated further research in hyperbolic metamaterials as a means to beating the diffraction limit and presented a proof of concept of a semiconductor based device capable of sub-diffraction and sub-wavelength

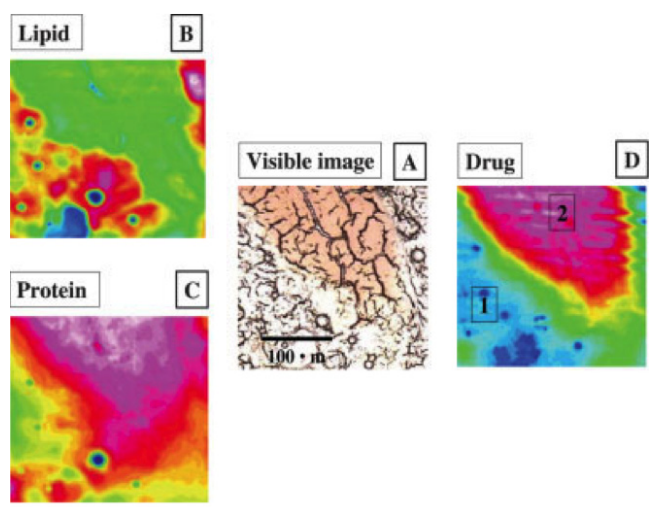

FIG. 12. Shown: utilizing spectroscopy and microscopy allows for drug permeation within tissue to be observed. Figure from [13]. By employing sub-wavelength imaging techniques with HMMs in combination with spectroscopy, drug permeation within individual cells (cell dimensions are much smaller than that of tissue) may be obtained (nano-spectroscopy).
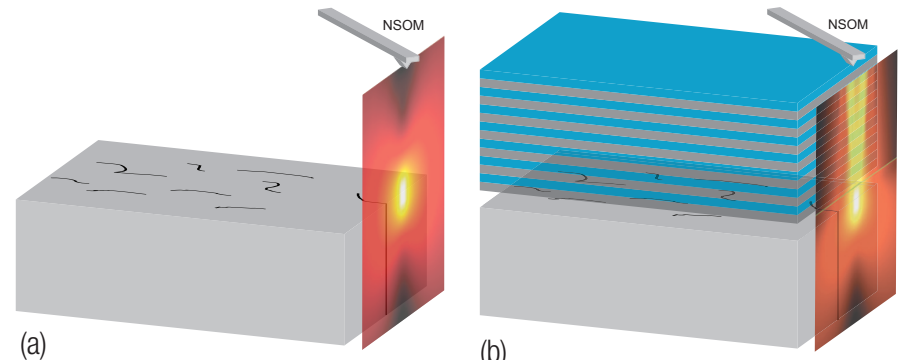

(b)

FIG. 13. Illumination of a Si wafer from below by $\lambda=9.8 \mu \mathrm{m}$ light. The incident light scatters through cracks in the wafer. (a) A sub-wavelength dimension crack in a Si wafer can be detected by a near field scanning optical microscope (NSOM) only if the detector is brought very close to the crack $(<\lambda / 6)$ (b) If a AllnAs/lnGaAs HMM is placed above the wafer, then the NSOM can detect the crack up to $2-3 \times \lambda$ from the wafer. A $10 \times$ increase in far field enhancement of high spatial frequencies can be acheived. 
imaging.

The necessary theory and electrodynamic formalism for describing the diffraction limit and sub-wavelength imaging was presented. Classical electrodynamics and the concept of Fourier optics was used to explain the conventional diffraction limit. Hyperbolic metamaterials, which have a hyperbolic dispersion and allow high spatial frequencies to propagate, were proposed as a solution to overcoming the diffraction limit.

Effective medium theory was developed for periodic multilayer structures and put forth as a design tool for hyperbolic metamaterials. It was shown that a multilayer stack of alternating layers of metal/dielectric displays hyperbolic metamaterial behaviour with a bulk extreme anisotropy: it behaves like a dielectric in one direction and a metal in another.

A hyperbolic metamaterial was then designed and demonstrated in the IR. Using a multilayer structure of AllnAs/ InGaAs (thickness 80/130.5 nm), with AllnAs as a dielectric and InGaAs as a customizable metal, it was shown that this multilayer structure exhibits bulk hyperbolic metamaterial behaviour in the IR region of wavlengths $\lambda=8.8$ to 10.5 $\mu \mathrm{m}$. Furthermore, the novel and exotic behaviour of the hyperbolic metamaterials was demonstrated: hyperbolic metamaterials capture evanescent near field waves and allow them to propagate to the far field. This was simulated for the effective medium slab of hyperbolic metamaterial and on the exact AllnAs/InGaAs multilayer structure using a transfer matrix technique. It was demonstrated that the bulk response of the exact multilayer model agrees very closely with that of the effective medium theory, therefore confirming the validity of the effective medium theory design technique for planar hyperbolic metamaterials.

The most difficult and also most important portion of the design was then completed. Namely, sub-wavelength imaging capabilities were demonstrated by showing a highly directional emission into the far field from a radiating point source near the effective medium AllnAs/InGaAs structure. The intricate and subtle details of the electric fields emitted by the point source was modeled through the Green's function formalism of EM sources. Potential devices and applications in pharmaceuticals and IC quality control were then proposed for this planar AllnAs/InGaAs hyperbolic metamaterial.

Future work will involve completing the last confirmation of the design: demonstrate sub-wavelength imaging capabilities by simulating the behaviour of a radiating point source above the exact multilayer structure. Moreover, experimental evidence must be obtained by fabricating and characterizing the AllnAs/InGaAs multilayer using molecular beam epitaxy.

\section{Acknowledgements}

This work was completed as a fourth year, Capstone Design Project in the department of Electrical and Computer Engineering. I would like to thank Dr. Chris Backhouse for instructing the course and providing useful feedback and direction throughout the course. My supervisor for the project was Dr. Zubin Jacob, an excellent teacher. Dr. Jacob provided the invaluable support and instruction required for understanding the complex electrodynamics and for me to enter into the field of metamaterials. In addition, I would like to thank my colleague, Sean Molesky for numerous insightful discussions.

\section{Reference}

[1] E. Hecht. Optics. Addison-Wesley, fourth edition, 2002.

[2] S. A. Campbell. Fabrication engineering at the micro and nanoscale. Oxford University Press, 2008.

[3] Z. Jacob, L. V. Alekseyev, and E. Narimanov. Optical Hyperlens: Far-field imaging beyond the diffraction limit. Optics express, 14(18):8247-56, September 2006.

[4] Z. Liu, H. Lee, Y. Xiong, C. Sun, nd X. Zhang. Far-field optical hyperlens magnifying sub-diffraction-limited objects. Science (New York, N.Y.), 315(5819):1686, March 2007.

[5] A. J. Hoffman, L. Alekseyev, S. S. Howard, K. J. Franz, D. Wasserman, V. a Podolskiy, E. E. Narimanov, D. L. Sivco, and C. Gmachl. Negative refraction in semiconductor metamaterials. Nature materials, 6(12):946-50, December 2007.

[6] G. R. Fowles. Introduction to Modern Optics. Dover, 1989.

[7] L. Novotny and B. Hecht. Principles of Nano-Optics, volume 54. Cambridge Univ Press, 2006.

[8] J. D. Jackson. Classical Electrodynamics. Wiley, third edition, 2007.

[9] T. Taubner, D. Korobkin, Y. Urzhumov, G. Shvets, and R. Hillenbrand. Nearfield microscopy through a SiC superlens. Science, 313(5793):1595, September 2006.

[10] P. Yeh. Optical Waves In Layered Media. Wiley, New York, 1988.

[11] S. A. Maier. Plasmonics (Fundamentals and Appli-cations). Springer, New York, 2007.

[12] T. Setala, M . Kaivola, and A. T. Friberg. Decomposition of the point-dipole field into homogeneous and evanescent parts. Physical Review, 59(1):1200-1206, May 1999.

[13] P. Garidel and M. Boese, Mid infrared microspectroscopic mapping and imaging: A bio-analytical tool for spatially and chemically resolved tissue characterization and evaluationof drug permeation within tissues. Microscopy Research and Technique, 70(4):336-349, 2007.

[14] A. Trigg. Applications of infrared microscopy to IC and MEMS packaging. IEEE Transactions on Electronics Packaging Manufacturing, 26(3):232-238, July 2003. 